Conclusions Gay men who report early FAI are at higher risk of HIV than those who report later FAI, and are more likely to have recently engaged in risky sexual behaviour. Discussion will focus on explaining these patterns and why health service providers need to pay attention to age at FAI.

\section{P2-S6.13 GAY MEN'S ASSESSMENT OF SEXUAL AND SOCIAL RISKS IN THE CONTEXT OF A RECENT HIV-POSITIVE DIAGNOSIS}

doi:10.1136/sextrans-2011-050108.365

\begin{abstract}
${ }^{1,2} \mathrm{D}$ Grace, ${ }^{2} \mathrm{M}$ Kwag, ${ }^{1} \mathrm{M}$ Steinberg, ${ }^{2} \mathrm{M}$ Rekart. ${ }^{1} \mathrm{BC}$ Centre for Disease Control, Simon Fraser University, Canada; ${ }^{2} B C$ Centre for Disease Control, University of British Columbia Canada
\end{abstract}

Background Technological innovations in HIV testing that allow for the diagnosis of very recently-acquired HIV infections provide opportunities to understand sexual and social risk perceptions before and after an HIV-positive diagnosis. Longitudinal interviews with a group of gay men who have received an early or acute HIV diagnosis represent an important opportunity to understand risk assessment beyond individual-level paradigms of risk analysis and to broaden our understanding of social and structural risk factors associated with HIV infection, diagnosis and disclosure.

Methods Study recruitment is being conducted through six clinical sites in British Columbia, Canada (April 2009-December 2012) by the CIHR Team in the Study of Acute HIV Infection in Gay Men. Participants ( $\mathrm{n}=12$ at time of analysis) completed a series of selfadministered questionnaires and semi-structured face-to-face interviews. Baseline qualitative interviews were recorded, transcribed verbatim and analysed. A thematic analysis, informed by a social organization of knowledge perspective, was conducted.

Results Three interrelated domains of risk assessment emerged from the interviews. First, we explicate how men calculated the epidemiological or sexual risks of transmitting HIV before and after their diagnosis, and how such an assessment informed their sexual behaviours. Second, men described a myriad of experienced and perceived social risks, such as stigma and rejection, associated with the disclosure of their HIV-status to their family, friends, colleagues and intimate partners. Third, men identified potential problems with technologies of status notification which create a set of institutional risks related to the processes by which patients learn of their HIV-positive status. The relationship between these textuallymediated fields of risk is examined.

Conclusion A stratified conception of risk allows us to understand the everyday situations in which people assess HIV-related 'dangers' in their social and sexual lives. This formative research has important implications for educational campaigns on HIV transmission risk assessment for both HIV negative and positive gay men. This work can also inform counselling and support services to address how disclosure risks are negotiated during an early or acute HIV diagnosis. Important implications for clinical and public health practices, including how and when people are given their HIV diagnosis, are raised.

\section{P2-S6.14 SEXUAL BEHAVIOUR, VULNERABILITIES AND CLINICAL INTERVENTION AMONG MEN WHO HAVE SEX WITH MEN (MSM) ATTENDING STI CLINIC; STUDY FROM SOUTH INDIA}

doi:10.1136/sextrans-2011-050108.366

${ }^{1}$ V S Prasad, ${ }^{2}$ S Shekhar. ${ }^{1}$ Strategic Partnerships, Chennai, India; ${ }^{2}$ Swam, India

Introduction Timely uptake of clinical services by highly mobile MSM is pre-requisite to halt and reverse STI prevalence among communities in India. It is imperative to understand risk behaviour and vulnerable factors that lead to the spread of STI among communities. A study was undertaken to understand the sexual behaviour, vulnerabilities, among MSM attending STI clinic of the $\mathrm{CBO}$ at Kancheepuram District, Tamilnadu, India.

Method In the study 69 (72\%) clinic attendees for a period of 9 months were followed up and interviewed at the STI clinic within the project. Consent and commitment was taken from the clinical attendees to be enrolled in the study. The project counsellor administered the questionnaire followed by clinical examination by the project Clinical provider.

Results Among the 69 MSM, 75.3\%were 20-30 years \& 11\%were $31-40$ years, $4.3 \%$ were above 50 years. Majority were from lower socioeconomic strata of the society and consisted of illiterate people also. $10 \%$ were uneducated, $27 \%$ Graduates and $44 \%$ had $5-10$ th grade education. About one-third (23.2\%) were married heterosexually. $65 \%$ reported travel to other states and $88 \%$ reported travel to other districts during the period of study. Among these $70 \%$ reported unprotected anal sex with casual partners during travel. Sexual behaviour: During the study period more than two-third (75.3\%) had anal sex and all 69 MSM had oral sex. Condom usage, before and after counselling was $6 \%$ and $53.6 \%$ respectively. $15.9 \%$ had sex with female sex workers and $25 \%$ received money for sex. $43 \%$ MSM had sex only for pleasure and $32 \%$ have sex for both pleasure and money.

Conclusion It is imperative that the project develop specific programs like Partners meet or Lovers meet to introduce their partners to the project and thereby sensitise them on prevention of STI and increase condom use. Programs like "pre-departure counselling" about risks on unprotected casual encounters for MSM planning travel and "post- travel STI screening" should be attempted. Regular and periodic STI screening among MSM and partners may be implemented. This will help the project in addressing the risk of transmission of infections among the partners. Partners meet will be a platform for addressing greater Sexual behavioural issues beyond normative clinical interventions. Early diagnosis and Intervention of anal STI, regular Condom use and repeated Counselling are imperative to halt and reverse the STI epidemic among the MSM community.

\section{P2-S6.15 INVESTIGATING THE CRITICAL PROGRAMME COMPONENTS OF HIV PREVENTION PROGRAMME AMONG MSM-TS IN BANGALORE, KARNATAKA}

doi:10.1136/sextrans-2011-050108.367

R Prakash, S Isac, B M Ramesh, S Moses, R Washington, P Bhattacharjee, B Shetty, S Kumar. Karnataka Health Promotion Trust Bangalore, India

Background A number of studies in the context of HIV/STI epidemic and related vulnerability have focused on the risk reduction of HIV/STI prevalence and condom use among female sex workers (FSWs). However, such studies focusing the men having sex with men (MSM) and transgender remains limited. Since the risk reduction of HIV/STI vulnerability among MSM-T are equally important, a systematic study is required to identify the critical programme indicators which increases the condom use and reduces HIV/STI prevalence among MSM-Ts.

Methods Two rounds of IBBA data collected from the urban parts of the Bangalore district are used. Bi-variate analysis is used to crossclassify the outcome measures by those who are exposed to the programme than those who are not, whereas the application of binary logistic regression analysis is done to get the adjusted effect of programme indicators on condom related outcomes (condom breakage in past 1 month, zero unprotected sex acts with commercial clients in past 1 month, condom use at last sex with 